\title{
A Service Component-based Accounting and Charging Architecture to Support Interim Mechanisms across Multiple Domains
}

\author{
M. van Le, B.J.F. van Beijnum \\ Dept. of Computer Science \\ University of Twente \\ P.O. Box 217 \\ 7500 AE Enschede \\ The Netherlands \\ \{le,beijnum\}@cs.utwente.nl
}

\author{
G.B. Huitema \\ TNO Telecom/ \\ University of Groningen \\ P.O. Box 15000 \\ $9700 \mathrm{CD}$ Groningen \\ The Netherlands \\ g.b.huitema@telecom.tno.nl
}

Abstract

Today, telematics services are often compositions of different chargeable service components offered by different service providers. To enhance component-based accounting and charging, the service composition information is used to match with the corresponding charging structure of a service session. This enables the sharing of revenues among the service providers, and calculation of the total cost for the end-user. When multiple independent service providers are involved, it is a great challenge to apply interim accounting and charging during a service session in order to minimize financial risks between business partners. Another interesting development is the trend towards outsourcing accounting and charging processes to specialized business partners. This requires a decoupling between provisioning and accounting and charging processes. In this paper, we propose a comprehensive component-based accounting and charging architecture to support service session provisioning across multiple domains. The architecture, modeled in UML, incorporates an interim accounting and charging mechanism to enable the processing and exchange of accounting information needed to update intermediate charges for separate service components and the user's credit, even during the service provisioning phase.

\section{Keywords}

Accounting and charging, interim accounting, service components, multi-domain

\section{Introduction}

Under pressure to support rapid development and changes in business relationships, today's service providers are faced with many challenges in dealing with real-time accounting and charging. These challenges are due to a number of factors 
including: one-time relationships between end-users and service providers; onetime relationships between service providers; the character of the requested services; and the corresponding charging policy. New services such as e.g. internet conferencing, multimedia over broadband networks, multi-party calls and similar may result in sessions lasting for hours. Applying interim accounting and charging to update users/providers credit balance during service sessions can decrease the risk of revenue leakage [1]. The resulting decrease in financial risk can motivate service providers to engage in business.

Furthermore, interest is increasingly shifting away from flat rate charging towards usage based or event based charging [2], which brings additional complexity to accounting and charging management. Recent developments also show great demand for alternative accounting and charging architectures that can enhance service sessions across multiple domains. A number of important aspects are discussed below.

End-to-end significance - This aspect deals with the accounting and charging for service sessions as seen by the end-users. The users are interested in paying for the requested service as a whole, not in paying for separate service sessions that might be provided by several service providers in a single service session.

Real-time Accounting -The delivery of next generation services is characterized more than in the traditional world by speed, one-time relationships, anonymous users and providers. Real-time accounting and charging is a way to limit financial risks. Real-time operation covers both real-time accounting and realtime charging. In real-time accounting, usage records must be generated right after a service session or even during a service session. In real-time charging the same holds for the availability of charge records (or rated usage records) for billing or for interchanging with other related accounting systems.

Accounting by delegation - In order to concentrate on their core businesses, service providers should have the opportunity to outsource accounting and charging processes. Thus, accounting systems must be flexible and able to interoperate across domains. That is, an accounting system must be able to interoperate with service provisioning systems or/and other accounting systems situated in different domains.

In this paper, we propose an accounting and charging architecture to support service session composition across multiple domains. The proposed architecture also allows for outsourcing of accounting and charging processes. Further, we include interim accounting and charging mechanism as a means to minimize financial risks. We apply the Unified Modeling Language (UML) to model our component-based architecture in a generic way such that these components (e.g. classes) can be reused by various applications.

The remainder of this paper is organized as follows: Section 2 addresses related work in the area of accounting and charging management. Section 3 describes the business context model being the operational environment of our accounting and charging architecture. Section 4 discusses a service session information model. Section 5 outlines a conceptual accounting and charging model. 
Section 6 discusses some issues relating to accounting and charging policies. Section 7 illustrates how the proposed accounting and charging architecture can be applied in practice. Finally, Section 8 concludes the paper and discusses some aspects of our work in progress and future directions.

\section{Related work}

A large number of frameworks and architectures have been developed in the area of accounting, charging and billing management (in general understood as "accounting management" as a whole). This section indicates the position of our research in the context of related work.

The Telecommunications Information Networking Architecture Consortium (TINA-C) developed the TINA-C framework, in which service session management and accounting management are described in a distributed way [5]. However, the accounting architecture within TINA-C's framework is restricted and closely coupled to service provisioning conforming to TINA specification. It therefore inherently limits the possibilities for outsourcing accounting services [6]. The Telemanagement Forum (TMF) has defined e-TOM, the map of communication operation processes [7]. The decoupling of business processes onto functional areas fulfillment, assurance and billing as defined in e-TOM favors outsourcing of management processes to the so-called "process suppliers". We adopt the same philosophy since "accounting and charging" can also be outsourced. The project Market Managed Multiservice Internet (M3I) has also introduced a set of functional areas, namely: Provisioning Functions, Accounting Functions, and Business Policy Functions [8]. Since this classification of functional areas is generic, we adopt this classification and define our architecture on this basis.

Recently, the project FORM (part of the European fifth framework research program) delivered the "Inter-Enterprise Management" framework, in which multidomain accounting (e.g. federated accounting) is addressed [9]. FORM has adopted the division of functional areas of e-TOM. Nevertheless, FORM does not take interim accounting and charging into account. In [12] Radisic presents a generic service management model and an associated accounting management model where attention is paid to outsourcing possibilities of service components. However the outsourcing aspects of accounting management are not addressed. The Internet Engineering Task Force (IETF) working group Authentication, Authorization and Accounting (AAA) has defined an architecture (the $A A A$ architecture) for IP-services. In addition, the Diameter Protocol can also be incorporated into the AAA-architecture for the transport of accounting information $[10,11]$. Although interim accounting is mentioned in Diameter, to our knowledge, little attention has been paid among research communities to investigation of interoperability across accounting platforms with extension to interim accounting in more detail.

Our contribution presented in this paper is twofold: on the one hand, we propose a generic service component-based accounting and charging architecture that is flexible enough to accommodate multiple business models, including 
outsourcing opportunities; on the other hand, we show how interim accounting and charging records can be exchanged between the business partners during a service session in order to minimize financial risks to service providers.

\section{Business Context}

In this section we present the business context to a level of detail sufficient for capturing the multi-domain accounting and charging architecture. In the literature, several business models, several kinds of models have been introduced with varying levels of detail $[7,8,9]$. The constituent models that we present here broadly follow the proposal of Lewis in [13], which distinguishes, among others, Business Role Model and Business Process Model.

\subsection{Business Role Model}

The Business Role Model identifies different business roles and possible reference points. There seems to be little consensus about the fundamental roles, one of the reasons being that it highly depends on the domain of interest being modeled. TINA distinguishes five fundamental roles, whereas for instance M3I distinguishes about eleven fundamental roles [8]. In our model we use only three fundamental roles: user, service provider, and process supplier.

The user role subscribes to, uses and pays for services; the user role can only "consume" services and never "produce". The service provider role is capable of producing services; the provider role can also "consume" services provided by other service providers as and when they are needed to fulfill service provisioning obligations. The accounting \& charging supplier role is capable of handling accounting and charging processes delegated by service providers. The account supplier role is capable of managing users' accounts and the charging of end-to-end service sessions. In practice, an account supplier is the location where the user credit balance is actually processed. This can be done at a bank, a credit institution or in the simplest case at a service provider's site.

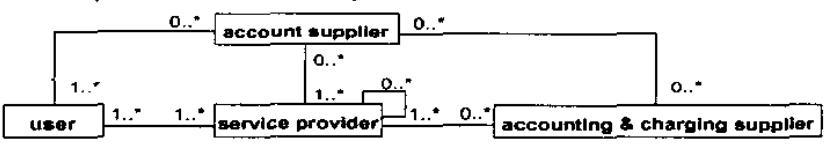

Figure 1: Business Role Reference Model

Figure 1 shows our business role reference model. Further specializations of the service provider role are possible. For instance, a connectivity provider and an information provider are specializations of the service provider role. In turn, an access provider and a backbone provider are for instance specializations of the connectivity provider. Similarly, a content provider and a market place provider are specializations of the information provider. 


\subsection{Business Process Model}

The provider role performs a set of business processes as to achieve its purpose. Many different processes can be identified. For instance, e-TOM identifies a set of processes organized in a TMN-like fashion. In our model, we take a much more focused view to highlight service usage (i.e. service sessions) and the accounting and charging thereof. The following three basic process sets are considered: provisioning process, accounting and charging process, and business policy process [8].

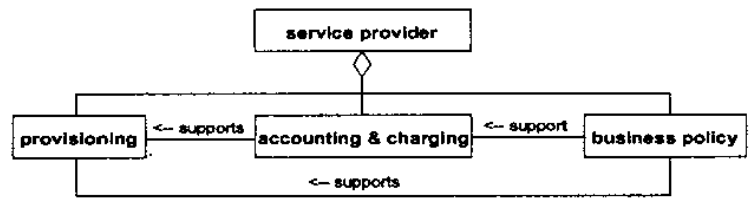

Figure 2: Relationship between a Business Role and the Function Sets

Figure 2 shows the relationship between a service provider role and the processes, modeled as an aggregation, not a composition. The rationale behind this way of modeling is that processes may be outsourced, or sub-processes may be delegated to other business roles. Provisioning depends on business policy, as business policy may determine provisioning strategies for instance when resource scarcity is experienced. Similarly, accounting and charging policies may be set to accommodate service dependencies, SLA dependencies and rating policies. Provisioning may depend on accounting \& charging in cases where (maximum) debit or (minimum) credit thresholds are set. Finally, accounting and charging depends on provisioning by virtue of the fact that usage records are to be received by the accounting function.

\section{Service Session Information Model}

In this section we present our Service Session Information Model in the context of the business model presented above. The key in this model is that a service session is shared knowledge between either a user-provider pair or a provider-provider pair. A multi-domain service session is modeled as a composition of service sessions. In our model we use the notion of service components, being an abstraction of resources used.

\subsection{User-Provider Service Session}

According to our approach, we gradually introduce and enhance the Service Session Information Model. As a starting point, we take the case in which there is a user and a single service provider as shown in Figure 3.

The association between the user and the service provider is a service session relationship, i.e. they are engaged in a service session in which the user has the requester role and the service provider the provisioner role. The service session 
class is the set of properties of the user - provider association, i.e. it is modeled as an association class. The service provider owns a set of service components, which is modeled as composition.

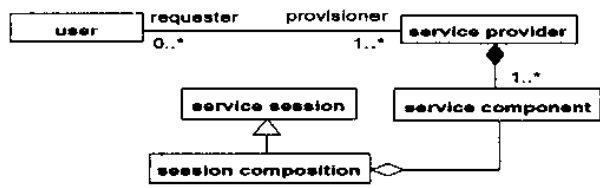

Figure 3: User-Provider Service Session Model

Indirectly, making service components available enables a service session. A service session defines the properties (e.g. contract) between the user and provider - the "what". How it is provided (by which service components, what arrangement) is internal to the provider - the "how". In order to clearly separate the "what" and "how", the session composition class is introduced. The session composition defined the "how" and is modeled as an aggregation of service components. Altogether, the session composition must inherit the properties of the service session it is supposed to provide; hence the service composition is modeled as a specialization of the service session. As a result, the user only "sees" the service session and only the provider knows how it is provided. The inheritance enforced by the specialization relationship enforces a tight coupling. The session composition class plays a vital role in the accounting and charging of service sessions.

\subsection{Provider-Provider Service Session}

In the next step of the model, we focus on the service session model between providers. We consider the provider - provider relationship as shown in Figure 4.

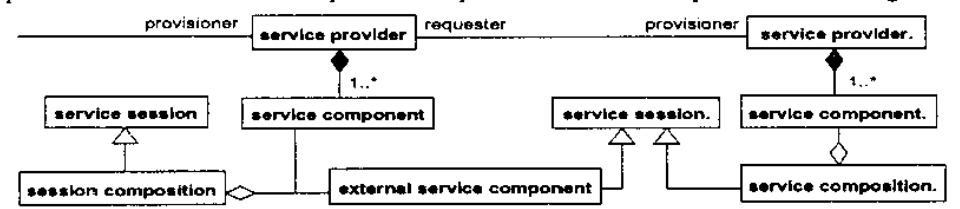

Figure 4: Provider-Provider Service Session Model

In this model, the set of properties of the provider-provider association is defined by the service session class. The external service component class represents a provider's internal view of this service session; this class is a specialization the service session class. In return, the external service component class is part of the service session composition, which in return is the specialization of the service session in which this provider participates as provisioner. So, the session composition class is the internal view of the service session in which the provider has a provisioner role, and the external service component is the internal 
view of a service session in which the provider has a requester role. The session composition class is defined as the aggregation of service components and external service components. The rule of composition is as follows: a session composition is the aggregate of at least one service component or external service component.

\section{Accounting and Charging Model}

In this section, we discuss a service session accounting and charging model. The model is derived from our business role reference model as described in Section 3. In order to focus on accounting and charging matters, we neglect the business policy function set in the discussion. The emphasis of our accounting and charging model is on the following:

- The information about service session composition, which is of vital importance in order to correlate accounting and charging information.

- The capability to exchange interim charge records between the providers during a service session.

- The user's account management, which is essential for interim accounting and charging mechanism.

- The possibility of outsourcing accounting and chatging responsibility to a process supplier.

Figure 5 shows an instance of the business role reference model together with the accounting and charging responsibility over three domains: user, service provider and account supplier. The service provider is responsible for the provisioning and accounting and charging of the requested service session. The service session charges are forwarded to the accounting supplier, who is responsible for managing the user credit (e.g. subtraction of session charges from the user credit). Figure 5 emphasizes the inter-domain relationship between two accounting \& charging classes belonging to separate domains.

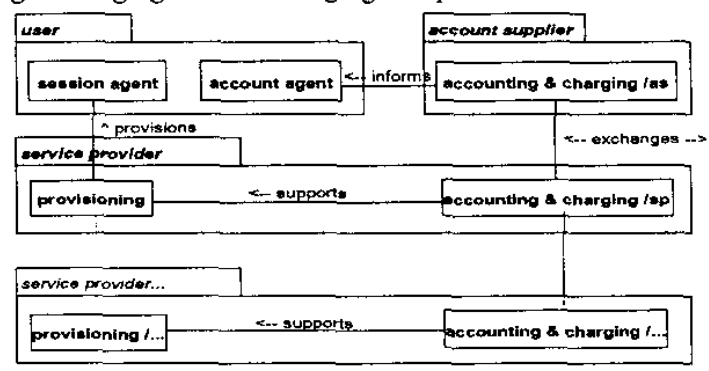

Figure 5: A Simple Accounting and Charging Model

Figure 6 depicts another instance of the business role reference model. In contrast to the previous model, here the service provider delegates accounting and charging responsibilities to the accounting \& charging supplier. Recursive relationships between service providers and accounting \& charging suppliers are 
possible. Therefore, a recursive association between multiple accounting \& charging classes is also possible. The responsibilities of different classes as described in our model are of generic nature, they can be organized in many possible ways within the solution space of our reference model (Figure 1) to support a multitude of possible business scenarios. We will illustrate this flexibility by means of an example in Section 7. In the following, we proceed to describe the constituent classes of the accounting and charging model in more detail.

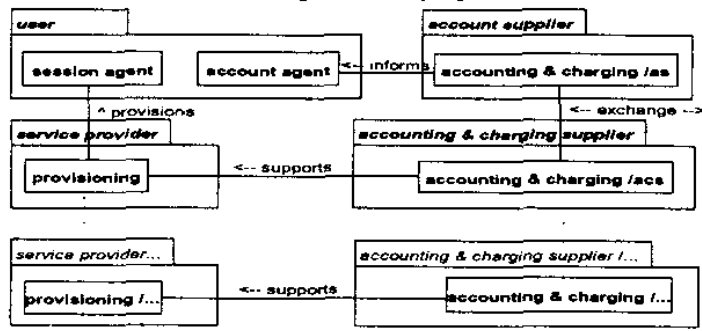

Figure 6: Delegation of Accounting and Charging

\subsection{User}

The user domain consists of two basic classes: session agent and account agent. The session agent is responsible for the request and control of a service session. This session agent may be some kind of application user interface. During a service session, actual user's credit information can be made available. The account agent is responsible for receiving and making this information noticeable. For instance, a user can consume a certain service, and at the same time observe his credit decreasing, thereby gaining more financial control. We do not model the relationship between the two classes in the user domain to express independence of their responsibility. For instance, the user can decide to turn off the functionality which allows for credit monitoring, but still can control and consume a service session.

\subsection{Service Provider}

The service provider domain consists of two basic classes: provisioning and accounting $\&$ charging. The provisioning is responsible for the fulfillment of requested service sessions. In general, the service provider provisions service sessions toward the requesters. This class can be an aggregation of other subclasses carrying different responsibilities, including: composing, provisioning and managing of service sessions (not shown in Figure 5 and 6).

The accounting \& charging class is responsible for the accounting and charging of service sessions within the service provider domain. This class supports the provisioning in order for service sessions to be provisioned. In our view, the relationship between provisioning and accounting \& charging (/sp) is 
tightly coupled. This means that service provisioning is only possible when it can be accounted and charged for.

\subsection{Accounting and Charging Supplier}

Here, we make a simplification of the reality by showing only one class, namely: the accounting \& charging (/acs). This class is responsible for the conducting of accounting and charging on behalf of a service provider. At a lower level of abstraction, the information related to service session composition and service usage must be communicated to accounting and charging supplier. Further, the accounting and charging supplier also needs to know who is to be claimed for the service charge. In this case, it is directly the account supplier, hence indirectly the user.

\subsection{Account Supplier}

The account supplier domain also embodies one class accounting \& charging (/ap). This class is responsible for managing the user credit. Under this responsibility, a number of tasks can be conducted. For instance, interim charge records must be subtracted from the user credit and the balance must be updated frequently. It is also the responsibility of accounting \& charging to trigger service session provisioning when user credit reaches a certain threshold.

Our approach advocates a general accounting \& charging class under the responsibility of an individual business domain. At a more detailed level, the tasks which must be done to carry out this responsibility within different domains may differ from one another.

\subsection{Details level of Accounting \& Charging - Service Provider}

The accounting \& charging within the service provider domain is an aggregation of different classes; each class is responsible for a number of specific tasks. Figure 7 depicts the aggregation of accounting \& charging, consisting of mediation, rating, accumulation and distribution. Before proceeding with this section, it is important to note that we distinguish two types of accounting records: usage records (URs) and charge records (CRs). In general usage records originate from service elements (switch, routers, event generator, etc.) and are not rated; whereas, charge records often originate from rating engines.

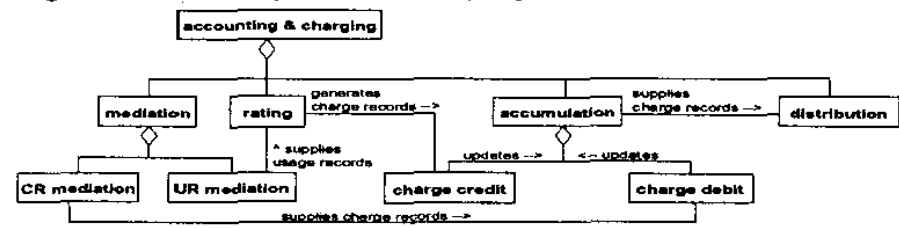

Figure 7: Accounting and Charging - A refined model for service provider

The mediation is an aggregation of $C R$ mediation and $U R$ mediation. The $C R$ mediation is responsible for collecting/receiving charge records originating from 
other service provider(s) related to eventual service request(s) of the service provider to compose its own service session. These charge records are already rated and can be supplied to the charge debit, whose responsibility is to keep track of the debit associated with the usage of requested service session(s). The $U R$ mediation is responsible for collecting usage records originating from service elements and supplying rating with usage records.

The rating is responsible for the application of the correct tariff on the usage records in order to generate charge records. These charge records are supplied to charge credit, whose responsibility is to keep track of the credit associated with the usage of service component(s) inside the service provider domain. A number of different rating techniques may be used. For the purpose of interim accounting and charging, rating engines are required to have high real-time performance in terms of throughput and processing $[15,16]$.

The accumulation is responsible for the correlation and merging of charge credit and debit of belonging to a provisioned service session. Moreover, correlation of different charge records is based on the information of service session composition including domain-unique service component identities, the identity of other business role to whom charge records must be sent, etc.

Finally, the distribution is responsible for forwarding the charge records to another service provider or process supplier. In our case, these are accounting \& charging supplier and account supplier.

\subsection{Details level of Accounting \& Charging - Account Supplier}

The accounting \& charging within the account supplier domain is an aggregation of a limited set of classes as shown in Figure 8, namely: CR mediation, charge debit, account management, and distribution. Most of these classes are discussed previously, with the exception of account management.

The account management is responsible for managing user credit balance. During a service session, it is necessary to monitor user credit balance, such that action can be taken if the balance reaches a certain pre-defined threshold. Further, the distribution is responsible for informing the user about the credit status and eventually, the total charge of a service session.

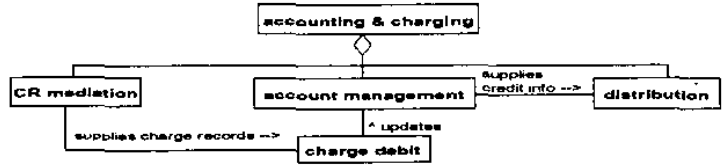

Figure 8: Accounting and Charging - A refined model for account supplier

\section{Accounting and Charging Policies}

So far, we have outlined some of the major components of the accounting and charging architecture. An important final aspect to discuss is the introduction of policies. Using policies in accounting and charging architectures has been 
proposed in other work, for instance in [3] a layered architecture has been introduced and policies are associated with each layer. In our architecture policies can be applied in a layered fashion as well; this permits a high degree of architectural flexibility.

Charge Accumulation Policy - As part of the business policy, the charge for a provisioned service session is to be computed from the charges for the constituents used for the provisioned service session. Different policies may be applied. Possible policies are:

- "Summation": the service session charge is the sum of the charges of the constituent charges

- "Weighted summation": each constituent charge is assigned a weight and the service session charge is the summation over the weighted charges.

Charge Exchange Policy - For the exchange of service session charges, the requester and provider roles may have agreed upon a specific policy. This policy may depend on e.g. the type of the service session, or other parameters considered relevant. As an illustration we consider a few possible policies. Here, we do not judge the technical, operational and business appropriateness.

- "Report any change of charge" policy: according to this policy, any change in the service session charge observed by the provider is reported to the requester.

- "Time-based" policy: according to this policy, charges are exchanged based on time, for instance governed by a timer.

- "Charge-increase" policy: according to this policy, charges are exchanged based on a certain amount of charge increase relative to the previous exchange.

- "Provider determined" policy: according to this policy, the service session provider determines the exchange policy. For example the provider may base the exchange on knowledge about the service session composition and internally known charging schemes. In case for instance the charge for content dominates the charge for transport, the provider may decide that charge increments incurred by the content charges govern the service session charge exchange.

User Account Policy - Users may have different types of accounts such as prepaid or postpaid. Also, user accounts may be treated differently depending on e.g. history. Furthermore, service specific user accounting rules may be set. User account policies also offer the potential for Customer Relationship Management (CRM), where individual users set service specific thresholds allowing end-users to have a higher level of service control.

\section{Ad-hoc Media Shop - A Use Case Scenario}

This section presents an application of our accounting and charging architecture. In the illustration, we emphasize the co-operation between business roles as they exchange accounting and charging information and show how accounting and charging processes collaborate in an end-to-end service session. 
Imagine the situation where a user travels frequently from one location to another. At a certain point in time, the user connects to a local wireless access network (e.g. hotspot) to retrieve multimedia content. To establish the session, the user makes a request to inter-Enterprise Service (IES) provider, indicating the specific content. The IES provider runs a verification procedure to check the user's credit worthiness (through the account supplier). In the case that the user credit is sufficient, the IES provider then requests the access provider and the media shop for an access session and a content session, respectively. The user service session is composed of an "enabling" service component (provided by the IES provider), an access service component, and a content component. Within the IES provider domain, the provisioning must communicate information about the service composition to the accounting \& charging/sp such that interim charges originated from the access provider and the media shop can be accumulated.

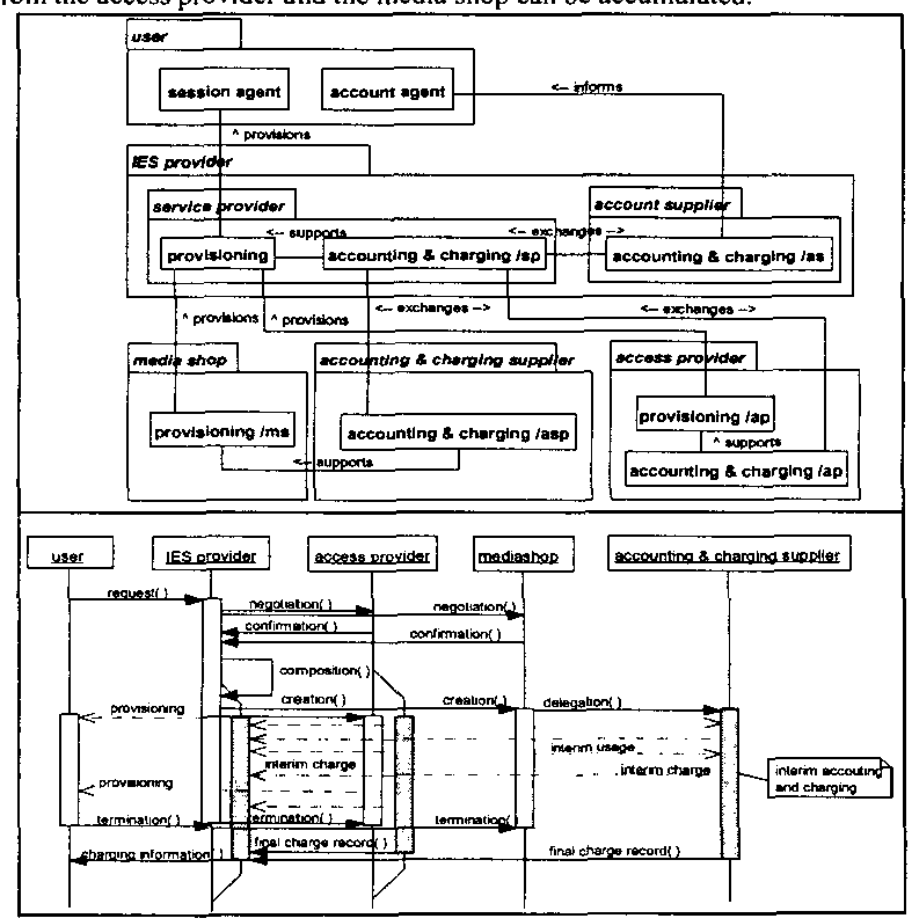

Figure 9: Interim Accounting and Charging of an Ah-hoc Media Service

The access provider is responsible for the accounting and charging of the access session requested by IES provider. During the provisioning phase, the access provider performs metering to supply UR mediation with interim usage 
records. Interim usage records are then rated into charge records. Next, the access provider forwards interim charge records to the IES provider.

The media shop decides to outsource accounting and charging processes, it therefore delegates all accounting and charging responsibility to the accounting \& charging supplier. During the provisioning phase, the accounting \& charging supplier collects interim usage records from the media shop, converts them into interim charge records, and then forwards interim charge records to the IES provider.

The IES provider is responsible for the accounting \& charging of user service sessions including user account management. It receives interim charge records from the access provider and the accounting \& charging supplier. The IES provider uses these charge records together with it own charge records to determine the intermediate charge of an user service session. Finally, intermediate charges are used to update the user credit balance accordingly. As an user service session terminates, both access provider and accounting \& charging supplier send a final charge record to the IES provider. This is to enable the finalization of accounting and charging for the service sessions.

Figure 9 illustrates the relationship between the business roles involved and the corresponding sequence diagram. The figure also illustrates how interim and final charge records can be exchanged between domains. Please note that this example is inspired by [9].

\section{Conclusion and Work in Progress}

In this paper, we have proposed a flexible service component-based accounting and charging architecture to enhance service session provisioning in multi-domain environments. The architecture is suitable to accommodate a great variety of business models. The architecture is component-based, in which classes are defined in a generic way in order to favor the reuse of the components in the design of accounting and charging systems. The introduction of interim usage record and charge record opens possibilities to investigate real-time operation of accounting and charging during a service session.

We are currently working on the specification of the information exchange between provisioning and accounting \& charging classes. The information is based on the Service Session Information Model as presented in Section 4. In addition, we are investigating the near real-time performance aspect of accounting and charging systems derived from the architecture using the simulation tool Network Simulator 2 [17]. Furthermore, study on the applicability of our architecture to web-services is also part of our future work.

\section{Acknowledgement}

The authors would like to thank Dr. Ing Widya, Erwin Molendijk and Lin Li for the fruitful technical discussions. Further, the authors wish to thank Dr. Val Jones and the anonymous reviewers for their comments and suggestion to improve the quality of the paper. 


\section{References}

[1] Kabira Technologies, Inc., "Analysis of Revenue Leakage in Convergent Service Networks and Convergent Transaction Processing", 2002.

[2] F. Ghys, A. Vaaraniemi, Component-Based Charging in a Next-Generation Multimedia Network, World Telecommunication Congress (WTC2002), September 2002.

[3] T. Zseby, S. Zander, G. Carle, "Policy-Based Accounting", RFC 3334, October 2002.

[4] IPDR organization, "Network Data Management - Usage For IP-based Services", version 3.1.1, October 2002.

[5] T. Hamada, "Accounting Management Architecture", version 1.3, TINA-C, 1996.

[6] P. Hellemans, Cliff Redmond, Koen Daenen, Dave Lewis, "Accounting Management in a TINA-Based Service and Network Environment", Proceeding of the 6th International Conference on Intelligence in Services and Networks, Barcelona, Spain, April 1999.

[7] TMForum: eTOM: The Business Process Framework for the Information and Communication Services Industry, TM Forum Approved/Version3.0, June 2002 (www.tmforum.org).

[8] M3I: Requirements Specification Reference Model, deliverable 1, July 2000 (http://www.m3i.org).

[9] B. Bhushan, E. Laray, "Federated Accounting: Service Charging and Billing in a Business-to-Business Enviroment", IFIP/IEEE International Conference on Management of Multimedia Networks and Services, Santa Barbara, CA, USA. October 2002.

[10] C. de Laat, G. Gross, L. Gommans, J. Volibrecht, D. Spence, "Generic AAA Architecture", RFC 2903, August 2000.

[11] P. Calhoun, J. Loughney, E. Guttman, G. Zorn, J. Arkko, "Diameter Base Protocol", < draft-ietf-aaa-diameter-13>, October 2002.

[12] I. Radisic, "Using Policy-Based Concepts to Provide Service Oriened Accounting Management", IEEE/IFIP Intemational Symposium on Network Operation and Managenet, Florence, Italy, April 2002.

[13] D. Lewis, V. Wade, B. Cullen, "Towards the Technology Neutral Modelling of Management Components", Journal of Network and Systems Management, vol. 11, No. 1, 2003.

[14] Brazil, Eamonn de Leastar, Conor Ryan, Mícheál Ó Foghlú, “ Workbook Approach to Algorithm Design and Service Accounting in a Component Orientated Environment", Waterford Institute of Technology, 2002.

[15] Highdeal, http://www.highdeal.com/.

[16] M. van Le, B.J.F. van Beijnum, B.L. de Goede, "Real-time Service Accounting", IEEE Workshop on IP Operations and Management, Dallas, TX, USA, October 2002.

[17] NS Home Page, http://www.isi.edu/nsnam/ns/. 